\title{
Irrigação no cultivo orgânico de alface utilizando Irrigás, tanque Classe A e um sistema automático de baixo custo
}

\author{
Irrigation with Irrigás, Class A pan and an low cost controller in the organic \\ cultivation of lettuce
}

\begin{abstract}
Fabíola Vieira Gonçalves ${ }^{I}$ Leonardo Oliveira Medici ${ }^{I I}$ Wilk Sampaio de Almeida ${ }^{\text {III }}$ Daniel Fonseca de Carvalho ${ }^{\mathrm{III}}$ Hugo Thaner dos Santos ${ }^{\mathrm{IV}}$ Daniela Pinto Gomes ${ }^{\mathrm{III}}$
\end{abstract}

\section{RESUMO}

O presente estudo teve como objetivo comparar um sistema automático de baixo custo a outras duas técnicas de manejo (sistema Irrigás e tanque classe A) na cultura da alface (Lactuca sativa L.) com relação à operacionalidade dos sistemas, custo dos equipamentos, lâminas aplicadas e dados de produção da cultura. O cultivo foi desenvolvido num delineamento experimental de blocos ao acaso, com quatro tratamentos casualizados em seis blocos. Os tratamentos foram caracterizados pelo sistema Irrigás, tanque Classe A (TCA) e pelo uso de um acionador automático de baixo custo para irrigação (AAI), em duas tensões de água no solo (9,0kPa e 12,0kPa). Foram avaliados dados de lâmina aplicada, eficiência no uso da água e as variáveis de produção, área foliar, massa fresca e seca da parte aérea, diâmetro e altura da cabeça. Não houve diferença significativa entre os tratamentos para nenhuma das variáveis de produção avaliadas $(P>0,05)$ e as lâminas totais referentes aos diferentes métodos de manejo foram próximas à evapotranspiração de referência (ETo) acumulada no período. A eficiência no uso da água nos tratamentos TCA, AAI/9kPa, Irrigás, AAI/12kPa foi, respectivamente, 241,4; 217,9; 205,5 e 267,1 $\mathrm{kg} \mathrm{ha}^{-1}$ $\mathrm{mm}^{-1}$, enquanto as produtividades foram respectivamente, 32,80; 28,86; 28,80 e 35,45Mg ha . $^{-1}$ Os resultados obtidos são próximos de valores reportados na literatura, permitindo concluir que as metodologias de manejo avaliadas foram eficientes para o cultivo de alface, e que, dessa forma, a escolha do método dependerá da disponibilidade de mão-de-obra e também do nível de treinamento do agricultor.

Palavras-chave: manejo da irrigação, automação, Lactuca sativa $L$.

\section{ABSTRACT}

The objective of the present research was to compare an low cost controller with two other techniques of irrigation

\begin{abstract}
management, Irrigas system and Class A pan, in the cultive of lettuce (Lactuca sativa L.), concerning operability of systems, equipment costs, applied depths and crop yield data. The cultivation was developed in a randomized complete blocks design with four treatments randomized in six replicates. The treatments were characterized by the system Irrigás, Class A pan (TCA) and the use of automatic irrigation of low cost device (AAI) in two different soil water tensions $(9.0 \mathrm{kPa}$ and $12.0 \mathrm{kPa}$ ). Data of irrigation depth, water use efficiency and production variables leaf area, fresh and dry weight of shoots, diameter and height of the head were evaluated. There wasn't significant difference between treatments for any of the production variables evaluated $(P>0.05)$ and total irrigation depths were related to different management practices and close to the evapotranspiration estimated reference (ETo) accumulated in the period. The water use efficiency in treatments TCA, AAI/9kPa, Irrigás, AAI/12kPa were, respectively, 241.4, 217.9, 205.5 and $267.1 \mathrm{~kg} \mathrm{ha}^{-1} \mathrm{~mm}^{-1}$, while the productivities were, respectively, 32.80, 28.86, 28.80 and $35.45 \mathrm{Mg} \mathrm{ha}^{-1}$. The results obtained are close to values reported in the literature, allowing to conclude that the evaluated methodologies were efficient for growing lettuce and thus the method chosen would depend on the availability of skilled manpower and also of the level of farmer training.
\end{abstract}

Key words: irrigation management, automation, Lactuca sativa $L$.

\section{INTRODUÇÃO}

A crescente demanda por água, principalmente pelo setor agrícola (PIMENTEL et al., 1999; QADIR et al., 2003), tem proporcionado sérias preocupações quanto à sua disponibilidade

\footnotetext{
IDepartamento de Fitotecnia, Universidade Federal Rural do Rio de Janeiro (UFRRJ), 23894-850, Seropédica, RJ, Brasil. E-mail: fabiolaufrrj@gmail.com. Autor para correspondência.

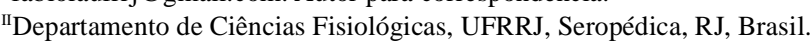

IIIDepartamento de Engenharia, UFRRJ, Seropédica, RJ, Brasil.

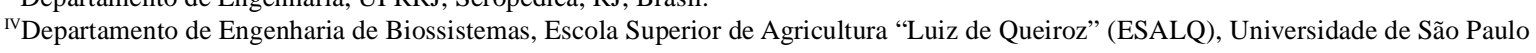
(USP), Piracicaba, SP, Brasil.
} 
para usos futuros. Nesse sentido, grandes esforços têm sido realizados por pesquisadores e agricultores a fim de garantir a qualidade e a disponibilidade deste recurso vital (MOHAMMAD et al., 2013).

De acordo com ROMERO et al. (2012), a aproximação mais racional para otimização da irrigação é o uso de controladores automáticos. Entretanto, na maioria dos casos, eles são operados por timers e não por sensores que expressam a demanda hídrica das plantas (MEDICI et al., 2010). Diversos autores têm estudado formas de automação de baixo custo para sistemas de irrigação baseados em disponibilidade de água do solo (PINMANEE et al., 2011; PRAKASH et al., 2012) e dados meteorológicos (ZHOU et al., 2009).

Em função de estudos desenvolvidos por nosso grupo de pesquisa, que vem demonstrando a funcionalidade do acionador automático de baixo custo para irrigação (AAI) (MEDICI et al., 2010; BATISTA et al., 2012; DIAS et al., 2013), desenvolveu-se este trabalho, no qual o AAI, que se baseia na tensão da água no solo, está sendo comparado a dois outros sistemas (tanque classe A e Irrigás), com relação à operacionalidade, custo, lâmina aplicada e produção de alface. O tanque é o método de manejo mais difundido e se baseia na estimativa da evapotranspiração a partir da evaporação de água, enquanto o Irrigás utiliza a tensiometria gasosa, ou seja, uma cápsula de cerâmica permite a passagem do ar quando a tensão da água ultrapassa um certo valor crítico (CALBO \& SILVA,2005).

\section{MATERIAL E MÉTODOS}

O estudo foi conduzido no município de Seropédica-RJ (Latitude 2248'00'S; Longitude 4341'00' W; altitude de 33,0m). O clima é do tipo Aw na classificação de Köppen. Durante o período experimental, as médias para temperatura e umidade relativa foram: $\mathrm{T}_{\text {máx }}=28,1^{\circ} \mathrm{C}$ e $\mathrm{T}_{\text {mín }}=16,0^{\circ} \mathrm{C}$; $\mathrm{UR}_{\max }=92,8 \%$ e $\mathrm{UR}_{\text {mín }}=45,4 \%$. A área experimental é caracterizada por Argissolo Vermelho-Amarelo, cuja caracterização química na camada de $0-10 \mathrm{~cm}$, mostrou teores de $\mathrm{P}=98\left(\mathrm{mg} \mathrm{dm}^{-3}\right) ; \mathrm{K}=135(\mathrm{mg}$ $\left.\mathrm{dm}^{-3}\right) ; \mathrm{Ca}=4,5\left(\mathrm{mg} \mathrm{dm}^{-3}\right) ; \mathrm{Al}=0\left(\mathrm{cmol}_{\mathrm{c}} \mathrm{dm}^{-3}\right) ; \mathrm{Mg}=$ $1\left(\mathrm{cmol} \mathrm{dm}^{-3}\right)$ e na camada de $0-20 \mathrm{~cm}^{\mathrm{c}}$ teores de $\mathrm{P}=$ 140,3 (mg dm$\left.{ }^{-3}\right) ; \mathrm{K}=101\left(\mathrm{mg} \mathrm{dm}^{-3}\right) ; \mathrm{Ca}=4,8\left(\mathrm{mg} \mathrm{dm}^{-3}\right)$; $\mathrm{Al}=0\left(\mathrm{cmol}_{\mathrm{c}} \mathrm{dm}^{-3}\right) ; \mathrm{Mg}=2,8\left(\mathrm{cmol}_{\mathrm{c}} \mathrm{dm}^{-3}\right)$. Para as mesmas camadas de solo, as curvas características (retenção) de água no solo são, respectivamente:

$$
\theta_{0-10}=0,113+\frac{0,939}{\left[1+\left(1,779 . \Psi_{\mathbb{m}}\right)^{3,2654}\right]^{0,1878}}
$$

$\theta_{10-20}=0,133+\frac{0,910}{\left[1+\left(1,185 \Psi_{m}\right)^{8,9664}\right]^{0,1754}}$

Antes da implantação do experimento, houve o preparo convencional do solo com posterior encanteiramento. A adubação foi realizada com esterco bovino na dose equivalente de $10 \mathrm{Mg} \mathrm{ha}^{-1}$. As mudas de alface foram transplantadas no dia 14 de agosto de 2012 (29 dias após a semeadura- DAS), com espaçamento entre linhas e plantas de $0,25 \mathrm{~m}$, em canteiros com 1,0m de largura e 3,0m de comprimento. Aos 20 dias após o transplantio, foi realizada uma adubação de cobertura com torta de mamona (5\% de $\mathrm{N}$ ), em linha de plantio, a uma dosagem de $50 \mathrm{~kg} \mathrm{ha}^{-1}$ de N. Durante o ciclo da cultura, capinas manuais eram realizadas sempre que necessárias.

O cultivo da alface (cultivar 'Regina') foi desenvolvido a campo em delineamento experimental em blocos ao acaso, com quatro tratamentos casualizados em seis blocos, totalizando 24 parcelas experimentais, com área útil de 16 plantas centrais de cada parcela. Os tratamentos foram caracterizados por diferentes métodos de manejo da irrigação (tanque Classe A, Irrigás e Acionador Automático com duas regulagens). Nos primeiros 13 dias após o transplantio (DAT), utilizou-se o sistema de irrigação por aspersão e, em seguida, o sistema por gotejamento, no qual o volume de água aplicado foi monitorado por meio de hidrômetros (alfa mnf, FAE Tecnologia, Fortaleza) instalados no início das linhas de derivação referentes a cada tratamento. Para o cálculo das lâminas, considerou-se o fator de localização $\left(\mathrm{k}_{\mathrm{L}}\right)$, no qual foi utilizado o percentual de área sombreada (KELLER \& BLIESNER, 1990).

O manejo pelo método do tanque Classe A (TCA), instalado próximo à área experimental, foi realizado de acordo com ALLEN et al. (1998), a partir das leituras diárias de evaporação no tanque (Ev).

$$
\mathrm{ETo}=\mathrm{Ev} \cdot \mathrm{kp}
$$

em que, ETo = evapotranspiração de referência $(\mathrm{mm}$ $\left.\operatorname{dia}^{-1}\right) ; \mathrm{Ev}=$ evaporação do tanque Classe $\mathrm{A}\left(\mathrm{mm} \mathrm{dia}^{-1}\right)$; $\mathrm{e} \mathrm{kp}=$ coeficiente do tanque.

A evapotranspiração da cultura (ETc) foi determinada multiplicando a ETo pelos coeficientes de cultivo da alface (kc) em função de cada fase de desenvolvimento da cultura (0,7 inicial; 0,85 vegetativo; 1,0 produção e 0,95 maturação) (MAROUELLI et al., 1996; ALLEN et al., 1998), os quais foram corrigidos, conforme metodologia também apresentada por ALLEN et al. (1998).

Para o manejo pelo sistema Irrigás, foram adotados três conjuntos de sensores de $15 \mathrm{kPa}$, sendo cada um deles composto por duas cápsulas: uma denominada de raiz e outra denominada de controle. 
Estas foram instaladas perpendicularmente à linha de plantio, na profundidade de $0,2 \mathrm{~m}$ (raiz) e $0,4 \mathrm{~m}$ (controle), aos 13 DAT. Os sensores foram instalados abaixo do emissor gotejador e suas leituras eram realizadas diariamente no período da manhã (por volta de 9h). A irrigação era efetuada quando pelo menos dois dos sensores indicavam leitura: SECO. A determinação do volume de água a ser aplicado era realizada por tentativas. Sendo assim, transcorrido o período de uma hora da irrigação, as leituras nos sensores eram refeitas e, quando necessário, maior quantidade de água era aplicada.

Dos tratamentos em estudo, dois constituíam-se no uso do Acionador Automático para Irrigação (AAI), instalados de modo a proporcionar tensões máximas de água no solo de $9 \mathrm{kPa}(\mathrm{AAI} / 9)$ e $12 \mathrm{kPa}(\mathrm{AAI} / 12)$. As diferentes tensões eram obtidas pelo desnível entre o pressostato e sensor (MEDICI et al., 2010), os quais foram, respectivamente, de 0,9 e 1,2m. A instalação dos acionadores ocorreu aos 13 DAT, sendo os sensores posicionados horizontalmente sob a linha de gotejamento, mais precisamente abaixo de um gotejador e paralelamente à linha de cultivo. $\mathrm{O}$ aporte de energia foi realizado por baterias $12 \mathrm{~V}$. Em ambos os tratamentos, o sensor de tensão foi instalado a $0,2 \mathrm{~m}$ de profundidade. Cada tratamento era representado por um conjunto de três acionadores, instalados em diferentes blocos. A instalação elétrica foi realizada de modo a permitir a irrigação de toda a área do tratamento quando um dos acionadores demandasse irrigação. Assim, a água era aplicada de forma automática sempre que a tensão da água no solo atingisse os valores correspondentes à $9 \mathrm{kPa}$ e $12 \mathrm{kPa}$, de acordo com o tratamento. A instalação dos acionadores ocorreu aos 13 DAT, sendo os sensores posicionados horizontalmente sob a linha de gotejamento, mais precisamente abaixo de um gotejador e paralelamente à linha de cultivo. $\mathrm{O}$ aporte de energia foi realizado por baterias $12 \mathrm{~V}$.

Os dados meteorológicos utilizados para análise e discussão dos resultados foram obtidos junto à estação automática do INMET, localizada no município de Seropédica/RJ.

A colheita foi realizada no dia 21/09/2012, sendo segmentada em duas partes: inicialmente, foram colhidas 12 plantas para as mensurações de massa fresca e seca, diâmetro, altura e número de folhas; as quatro plantas restantes foram utilizadas para medição da área foliar. Apenas a parte aérea (cabeça comercial) foi avaliada.

A eficiência do uso da água (EUA) foi obtida pela razão da massa fresca total da cultura da alface pela lâmina total aplicada para cada tratamento, conforme a equação:

$$
\operatorname{EUA}\left(\mathrm{kg} \mathrm{ha}^{-1} \mathrm{~mm}^{-1}\right)=\frac{\mathrm{P}}{\mathrm{I}+\mathrm{Pa}}
$$

em que a $\mathrm{P}$ é produtividade da cultura $\left(\mathrm{kg} \mathrm{ha}^{-1}\right)$; I a lâmina aplicada pela irrigação (mm); e Pe a precipitação efetiva $(\mathrm{mm})$. Para o cálculo das lâminas (I), considerou-se a área irrigada referente a cada método de manejo, fazendo-se a devida correção pela porcentagem da área sombreada (PAS), obtida por meio de observações a campo ao longo do ciclo de desenvolvimento da cultura. Foi considerada como precipitação efetiva $(\mathrm{Pe})$ a fração da lâmina de água precipitada $(\mathrm{mm})$, correspondente a um valor menor ou igual à evapotranspiração da cultura, referente a um turno de rega de dois dias.

Os resultados de massa fresca e seca, diâmetro, altura e número de folhas e área foliar foram submetidos à análise de variância, com teste $\mathrm{F}$ a $5 \%$ de probabilidade.

\section{RESUTADOS E DISCUSSÃO}

$\mathrm{O}$ aporte de água à cultura por meio da precipitação totalizou $13,65 \mathrm{~mm}$ no período de avaliação. Com a irrigação por aspersão, foram aplicados $32,91 \mathrm{~mm}$ e, por gotejamento, diferentes lâminas foram aplicadas para as distintas formas de manejo: 78,3mm (TCA), 82,6mm (Irrigás 15kPa), $74,9 \mathrm{~mm}$ (AAI/9kPa), e 75,1 mm (AAI/12kPa). As lâminas totais referentes aos diferentes métodos de manejo estão próximas à ETo acumulada no período, que totalizou 128,8mm (Figura 1). Adotando o manejo pelo AAI com 9kPa, BATISTA et al. (2013) contabilizaram uma lâmina total para a cultura da alface, correspondente a $89 \%$ da ETo. No presente estudo, o valor percentual equivalente da lâmina total aplicada no mesmo tratamento foi de $94,3 \%$ da ETo.

O fator água, como fonte de variação, vem sendo amplamente estudado, objetivando-se buscar a lâmina economicamente viável na produção agrícola comercial. HAMADA \& TESTEZLAF (1995), em estudo com diferentes lâminas de irrigação no cultivo de alface, encontraram valores de massa fresca entre $149 \mathrm{~g}$ e $224 \mathrm{~g}$ por planta. Esses valores corroboram os encontrados neste trabalho, no qual as médias dos tratamentos variaram de 180 a $221 \mathrm{~g}$ por planta (Tabela 1). BATISTA et al. (2013), utilizando o AAI na tensão de $9 \mathrm{kPa}$, encontraram massa fresca de $301,8 \mathrm{~g}$ por planta e massa seca de $10,9 \mathrm{~g}$ por planta. Com relação à massa seca, o valor obtido neste estudo (14,4g por planta) para o AAI $9 \mathrm{kPa}$ supera ao apresentado por BATISTA et al. (2013). Em estudo com mudas de mamoeiro, utilizando-se o mesmo 


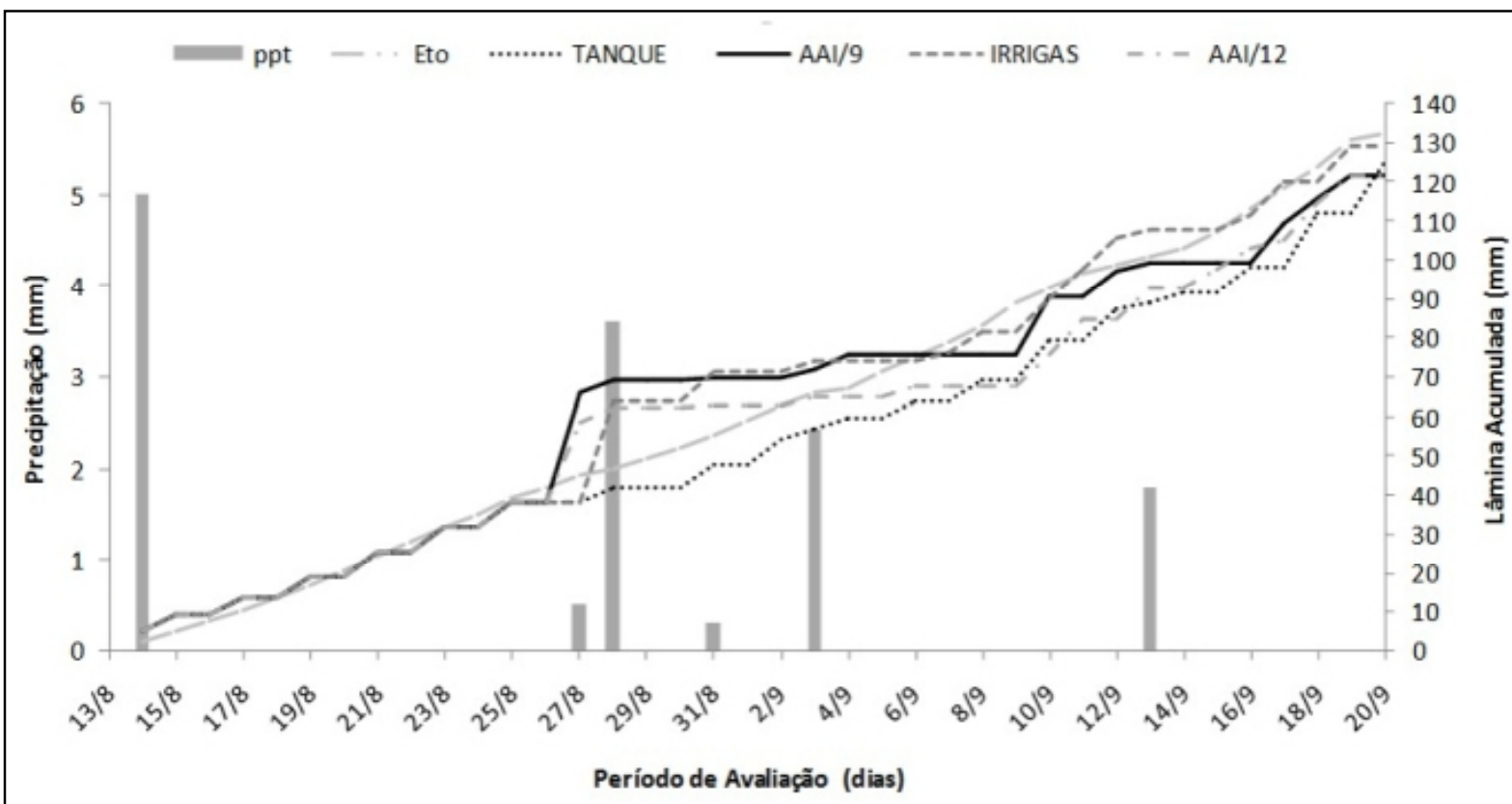

Figura 1 - Precipitação - ppt(mm) e lâminas totais acumuladas $(\mathrm{mm})$ referentes às diferentes técnicas de manejo da irrigação (TANQUE tanque Classe A; AAI/9 e AAI/12 - Acionador Automático para Irrigação nas tensões de 9 e 12kPa, respectivamente; IRRIGAS - Irrigás 15kPa), ao longo do cultivo da alface (ETo - Evapotranspiração acumulada no período), Seropédica, 2012.

acionador (AAI), DIAS et al. (2013) observaram que a tensão de $9 \mathrm{kPa}$ proporcionou estresse hídrico na cultura. Esse fato indica que cada cultura necessita de uma disponibilidade adequada de água, a qual se relaciona diretamente com as variáveis de produção de interesse comercial (MEDICI et al., 2014).

Para massa fresca média e diâmetro, SALGADO et al. (2006) encontraram valores superiores às médias deste trabalho $(196,7 \mathrm{~g}$ por planta e $25 \mathrm{~cm}$, respectivamente), variando nos dois anos de estudo entre 284,2 e $343,9 \mathrm{~g}$ para massa fresca e de 30,7 a $35 \mathrm{~cm}$ para diâmetro.Com relação ao número de folhas, área foliar e altura de plantas, as médias nos tratamentos (34 a 36 folhas; 2510,2 a $2671,4 \mathrm{~cm}^{2}$; 20 a $20,3 \mathrm{~cm}$; respectivamente) foram superiores às observadas por outros autores (NEGREIROS et al., 2002; RADIN et al., 2004), em que as condições ambientais eram semelhantes. Os valores de produtividade $\left(32,8 ; 28,86 ; 28,8 ; 35,45 \mathrm{Mg} \mathrm{ha}^{-1}\right.$ para TAC, AAI/9, IRRIGAS e AAI/12; respectivamente) foram inferiores aos encontrados por SALGADO et al. (2006) (55Mg ha-1) e LEDO et al. (2000) $\left(45,5 \mathrm{Mg} \mathrm{ha}^{-1}\right)$ no cultivo a campo de alface (cultivar 'Regina 71'). Por outro lado, MAGGI et al. (2006) encontraram valores semelhantes aos apresentados, com produtividade máxima de $36,11 \mathrm{Mg} \mathrm{ha}^{-1}$.

Houve uma tendência de aumento da EUA com a redução na lâmina aplicada. Assim, o tratamento que apresentou a maior lâmina (Irrigás) foi também o que proporcionou a menor EUA $(222,95 \mathrm{~kg}$ $\left.\mathrm{ha}^{-1} \mathrm{~mm}^{-1}\right)$; seguido, em ordem crescente de EUA, dos manejos realizados pelo AAI/9 $\left(237,62 \mathrm{~kg} \mathrm{ha}^{-1} \mathrm{~mm}^{-1}\right)$, TCA $\left(262,59 \mathrm{~kg} \mathrm{ha}^{-1} \mathrm{~mm}^{-1}\right)$ e AAI/12 $\left(291,23 \mathrm{~kg} \mathrm{ha}^{-1}\right.$ $\mathrm{mm}^{-1}$ ). Resultados semelhantes foram encontrados por HAMADA \& TESTEZAF (1995) e ARAÚJO et al. (2010), que encontraram respostas decrescentes para EUA, à medida que maiores lâminas foram aplicadas. Em ambiente protegido, ARAÚJO et al. (2010) encontraram valores que variaram de 120 a $70 \mathrm{~kg} \mathrm{ha}^{-1} \mathrm{~mm}^{-1}$. MAGGI et al. (2006) obtiveram valores de EUA sob diferentes tensões de água no solo entre $205,9(28 \mathrm{kPa})$ a $368,5 \mathrm{~kg} \mathrm{ha}^{-1} \mathrm{~mm}^{-1}$ (35kPa). CUPPINI et al. (2010), realizando o manejo da irrigação em função de percentuais da evaporação do TCA em cultivo protegido, obtiveram EUA de 208,74; 187,30; $139,86 \mathrm{~kg} \mathrm{ha}^{-1} \mathrm{~mm}^{-1}$, respectivamente, para percentuais de 75,100 e $125 \%$.

Embora tenham proporcionado resultados semelhantes, os métodos de manejo da irrigação avaliados apresentam diferenças acentuadas quanto à operacionalidade. No sistema automático, a maior exigência ocorre na fase de montagem e instalação do equipamento, mas durante a sua condução a exigência de mão de obra é praticamente nula. Por outro lado, o tanque Classe A requer um conhecimento prévio dos dados meteorológicos e da evapotranspiração da cultura na região de cultivo, além de exigir leituras diárias de evaporação do tanque, assim como a sua 
Tabela 1 - Médias das variáveis de produção (MF- massa fresca; MS- massa seca; NF- número de folhas; AF- área foliar; altura e diâmetro das plantas) referentes às diferentes técnicas de manejo da irrigação na cultura da alface, Seropédica, 2012.

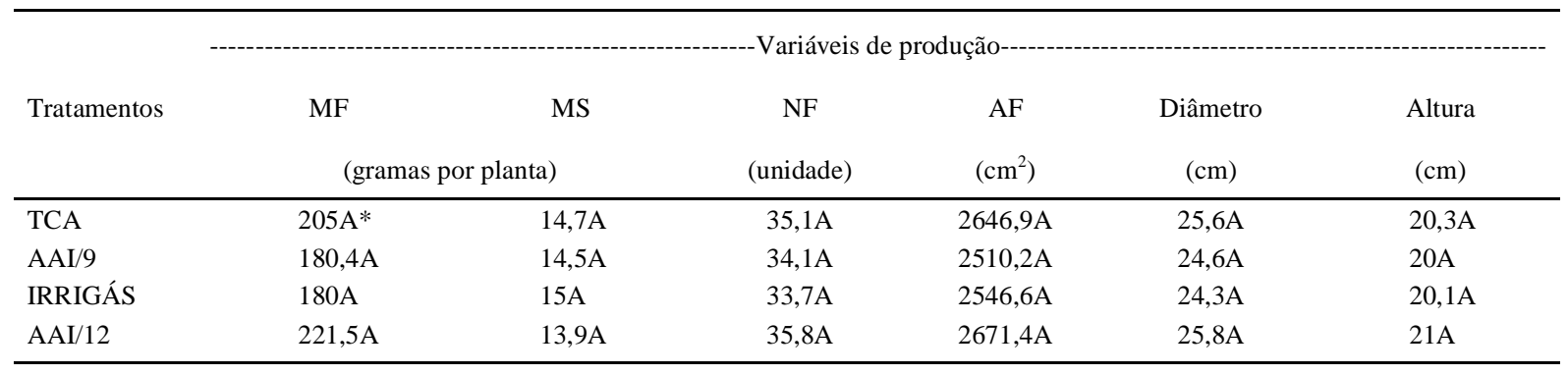

*Médias seguidas da mesma letra nas colunas não diferem significativamente pelo teste $\mathrm{F}$, a 5\% de probabilidade.

manutenção, quando necessário. $\mathrm{O}$ modelo do sistema Irrigás utilizado, com ajuste de lâminas por tentativa, é bastante simples de ser instalado e requer apenas que se defina a cultura e o sistema de irrigação que será utilizado para escolha da cápsula porosa mais adequada. No entanto, apresenta dificuldades no manejo, no que se refere ao ajuste da lâmina, pois exige que sejam feitas leituras após cada evento de irrigação, a fim de verificar se ela foi suficiente ou não, para que o sensor indique leitura ÚMIDO.

Além das diferenças operacionais, o custo do equipamento pode se constituir como decisivo para o agricultor na escolha do método de manejo a ser adotado. Dentre os métodos avaliados neste trabalho, o que apresenta maior custo é o tanque Classe A ( $\$ 3.877,00)$, seguido do Irrigás (R\$ 628,00), incluindo três conjuntos de sensores Irrigás PRO e um leitor (MPI-03) e, por fim, do AAI, com um custo aproximado de apenas $\mathrm{R} \$ 30,00$.

\section{CONCLUSÃO}

A comparação dos métodos demonstrou que existem diferenças com relação à operacionalidade e ao custo dos equipamentos, mas que estas não refletiram no desempenho da cultura, que não apresentou diferenças significativas $(\mathrm{P}>0,05)$ com relação às variáveis de produção da alface (massa fresca e seca, diâmetro e altura da cabeça e área foliar), assim como nas lâminas aplicadas, que totalizaram valores próximos à demanda evapotranspirométrica acumulada no período.

\section{AGRADECIMENTOS}

À Coordenação de Aperfeiçoamento de Pessoal de Nível Superior (CAPES) - Brasil (AUXPE - PNPD 02779/09), pelo apoio financeiro prestado.

\section{REFERÊNCIAS}

ALLEN, R.G. et al. Crop evapotranspiration: guidelines for computing crop water requirements. Irrigationand Drainage, 1998. 301p. (Paper, 56). Disponível em: <http://www.fao.org/ docrep/x0490e/x0490e00.HTM>. Acesso em: 22 ago. 2013.

ARAÚJO, W.F. et al. Rendimento e eficiência do uso da água pela alface em função da lâmina de irrigação. Revista Caatinga, v.23, n.4, p.115-120, 2010. Disponível em: <http://periodicos.ufersa. edu.br/revistas/index.php/sistema/article/view/1824>. Acesso em: 22 ago. 2013.

BATISTA, S.C.O. et al. Production of automatically watered lettuce with a low cost controller. Journal of Food, Agriculture \& Environment, v.11, n.2, p.485-489, 2013. Disponível em: <http:// world-food.net/production-of-automatically-watered-lettuce-witha-low-cost-controller/>. Acesso em: 22 ago. 2013.

CALBO, A.G.; SILVA, W.L. de C e. Sistema irrigas para manejo de irrigação: fundamentos, aplicações e desenvolvimentos. Brasília, DF. Embrapa Hortaliças, 174p. 2005. Disponível em: <http://www. cnph.embrapa.br/paginas/serie_documentos/publicacoes2006/ sistema_irrigas.pdf $>$. Acesso em: 21 jan. 2014.

CUPPINI, D.M. et al. Efeito da irrigação na produção da cultura de alface (Lactuca sativa L.), variedade "pira roxa", manejada através de "tanque Classe A" em ambiente protegido. Revista Perspectiva, v.34, n.127, p.53-61, 2010. Disponível em: <http:// www.uricer.edu.br/new/site/pdfs/perspectiva/127_121.pdf >. Acesso em: 22 ago. 2013.

DIAS, C.C.O. et al. Papaya seedlings growth using a low-cost, automatic watering controller. Revista Brasileira de Fruticultura, v.35, n.2, p.040-046, 2013. Disponível em: <http://www.scielo.br/ pdf/rbf/v35n2/23.pdf>. Acesso em: 07 jan. 2014. doi: 10.1590/ S0100-29452013000200023.

HAMADA, E.; TESTEZLAF, R. Desenvolvimento e produtividade da alface submetidas a diferentes lâminas de água através da irrigação por gotejamento. Pesquisa Agropecuária Brasileira, v.30, n.9, p.1201-1209, 1995. Disponível em: <http://seer.sct. embrapa.br/index.php/pab/article/view/4415>. Acesso em: 22 ago. 2013.

KELLER, J.; BLIESNER, R.D. Sprinkle and trickle irrigation. New York: Avibook, 1990.649 p. 
LEDO, F.J.S. et al. Desempenho de cultivares de alface no Estado do Acre. Horticultura Brasileira, v.18, n.3, p.225-228, 2000. Disponível em: <http://www.scielo.br/scielo.php?script=sci_ arttext\&pid=S0102-05362000000300017\&lng=en\&nrm=iso $>$. Acesso em: 07 set.2013. doi: 10.1590/s0102-05362000000300017.

MAGGI, M.F. et al. Produção de variedades de alface sob diferentes potenciais de água no solo em ambiente protegido. Irriga, v.11, n.3, p.415-427, 2006.

MAROUELLI, W.A. et al. Manejo da irrigação em hortaliças. Brasília: Embrapa-SPI: Embrapa-CNPH, 1996.72p.

MEDICI, L.O. et al. What about keeping plants well watered? Environmental and Experimental Botany, v.99, p.38-42, 2014 Disponível em: <http://www.sciencedirect.com/science/article/pii/ S0098847213001743>. Acesso em: 18 jan. 2014. doi: 10.1016/j. bbr.2011.03.031.

MEDICI, L.O.et al. Automatic controller to water plants. Scientia Agricola, v.67, n.6, p.727-730, 2010. Disponível em: <http:// dx.doi.org/10.1590/S0103-90162010000600016>. Acesso em: 22 ago. 2013. doi: S0103-90162010000600016.

MOHAMMAD, F.S. et al. Adoption of an intelligent irrigation scheduling technique and its effect on water use efficiency for tomato crops in arid regions. Australian Journal of Crop Science, v.7, n.3, p.305-313,2013.Disponível em: <http://www.cabdirect. org/abstracts/20133165191.html;jsessionid=F0A289C23C8149C5 F7C01794F765F7A5>.Acesso em: 07 set. 2013

NEGREIROS, M.Z.et al. Cultivares de alface em sistemas solteiro e consorciado com cenoura em Mossoró. Horticultura Brasileira, v.20, n.2, p.162-166, 2002. Disponível em: 〈http://www.scielo.br/ scielo.php?script $=$ sci_arttext $\&$ pid $=S 0102-05362002000200008>$. Acesso em: 07 set. 2013.doi: 10.1590/S0102-05362002000200008.

PIMENTEL, D.et al. Will limits of the earth's resources control human numbers? Environ. Sustainability, v.1, n.1, p.1939, 1999. Disponível em: <http://link.springer.com/article/ 10.1023/A:1010008112119>. Acesso em: 07 set. 2013. doi: 10.1023/A:1010008112119.

PINMANEE, S. et al. Development of a low-cost tensiometer driven irrigation control unit and evaluation of its suitability for irrigation of lychee trees in the uplands of Northern Thailand in a participatory approach. Journal of Horticulture and Forestry, v.3, n.7, p.226-230. 2011. Disponível em: <http://www. academicjournals.org/JHF/fulltext/2011/July/Pinmanee\%20 et\%20al.htm>. Acesso em: 07 set. 2013. doi: 10.1007/978-3-64233377-4_1

PRAKASH, et al. International Journal of Innovative Technology and Exploring Engineering, v.1, n.1, p.04-06, 2012. Disponível em: <http://www.ijitee.org/atTCAhments/File/v1i1/ A105051112.pdf $>$. Acesso em: 07 set. 2013.

QADIR, M. et al. Agricultural water management in waterstarved countries: challenges and opportunities. Agricultural Water Management. v.62, n.3, p.165-185, 2003. Disponível em: <http://www.sciencedirect.com/science/article/pii/S0378377 40300146X>. Acesso em: 07 set. 2013. doi: 10.1016/S03783774(03)00146-X.

RADIN, B. et al. Crescimento de cultivares de alface conduzidas em estufa e a campo. Horticultura Brasileira, v.22, n.2, p.178-181, 2004. Disponível em: <http://www.scielo.br/scielo.php?script=sci arttext\&pid=S0102-05362004000200003>. Acesso em: 07 set. 2013. doi: 10.1590/S0102-05362004000200003.

ROMERO, R. et al. Research on automatic irrigation control: State of the art and recent results. Agricultural Water Management, v.114, p.59-66, 2012. Disponível em: 〈http://www.sciencedirect. com/science/article/pii/S0378377412001746>. Acesso em: 07 set. 2013. doi: 10.1016/j.agwat.2012.06.026

SALGADO, A.S. et al.Consórcios alface-cenoura e alfacerabanete sob manejo orgânico. Pesquisa agropecuária brasileira, v.41, n.7, p.1141-1147, 2006. Disponível em: <http:// www.scielo.br/scielo.php?script $=$ sci arttext $\&$ pid $=S 0100$ 204X2006000700010>. Acesso em: 07 set. 2013.doi: 10.1590/ S0100-204X2006000700010.

ZHOU, Y. et al. A Wireless design of low-cost irrigation system using zigbee technology. In: INTERNATIONAL CONFERENCE ON NETWORKS SECURITY, Wireless Communications and Trusted Computing, 2009. p.572575. Disponível em: <http://ieeexplore.ieee.org/stamp/stamp. jsp?arnumber $=04908331>$. Acesso em: 07 set. 2013. doi: 10.1109/NSWCTC.2009.231. 